\title{
Effect of carrier system on the yield of human oocytes and embryos as assessed by survival and developmental potential after vitrification
}

\author{
J. Liebermann and M. J. Tucker \\ Georgia Reproductive Specialists, 5445 Meridian Mark Road Suite 270, \\ Atlanta, Georgia, 30342, USA
}

The present study was undertaken to examine the effect of a cooling regimen during vitrification on survival and morphological appearance of human oocytes after warming and the developmental potential of day 3 embryos. Aged human oocytes that had failed to fertilize and human embryos derived from abnormally fertilized zygotes that showed one pronucleus or three or more pronuclei were used as models in this study. In the first part of the study, 928 aged human oocytes that had failed to fertilize were vitrified. The viability of oocytes after vitrification using the hemi-straw system was slightly higher than it was using the cryoloop after warming $(85.4 \%(410$ of 480$)$ versus $80.6 \%$ (361 of 448$)$ ), but the difference was not significant. In the second part of the study, 266 embryos were vitrified. The survival of day 3 embryos after vitrification

\section{Introduction}

As early as 1985, ice-free cryopreservation of mouse embryos at $-196^{\circ} \mathrm{C}$ by vitrification was reported (Rall and Fahy, 1985) as a possible alternative approach to cryostorage. Many later studies were undertaken to reduce the time of the cryopreservation procedure, and also to try to eliminate the use of expensive programmable freezing equipment. One way to achieve these goals is to use vitrification techniques and protocols that allow oocytes and embryos to be placed in the cryoprotectant and then plunged directly into liquid nitrogen. The definition of vitrification is the solidification of a solution at a low temperature without the formation of ice crystals, by increasing the viscosity, using high cooling rates from 15000 to $24000^{\circ} \mathrm{C} \mathrm{min}-1$ (Fahy et al., 1984; Martino et al., 1996; Arav and Zeron, 1997; Vajta et al., 1997). The key aim of any vitrification technique and protocol is to achieve high rates of cooling in association with high concentrations of cryoprotectant. However, this is problematic and technically difficult to achieve (Rall, 1987). Cryoprotective agents are essential for the cryopreservation of cells. There is a practical limit of attainable cooling speed, and a

E-mail: jliebermann@t-online.de was improved by using a mixture of ethylene glycol and dimethyl sulphoxide in combination with the hemi-straw system method rather than the cryoloop method $(89.7 \%$ (122 of 136) versus $83.8 \%$ (109 of 130)), but the difference was again not significant. The potential for development up to compaction on day 4 in embryos vitrified using the hemi-straw system method was significantly higher $(37.7 \%)$ than it was in embryos vitrified using the cryoloop $\left(29.4 \% ; \chi^{2}, P=0.002\right)$. The hemi-straw system and cryoloop methods of vitrification are both successful, easy to perform, and demonstrate the ability of both carriers to vitrify different stages of development (oocytes and day 3 embryos). In addition, the success of both methods appears to be related to the rate of cooling and the lower concentration of cryoprotectant used. biological limit on the concentration of cryoprotectant tolerated by cells during vitrification. A critical concentration of cryoprotectant is required for vitrification; variation in concentration can lead to either osmotic or chemical toxicity. Therefore, a balance between maximixing the cooling rate and minimizing the cryoprotective concentration is important. The high concentration of cryoprotectant used for vitrification and the known biological and physicochemical effects of cryoprotectants make the toxicity of these agents a key limiting factor in cryobiology.

The present authors have found that a high cooling rate is an important factor in improving the effectiveness of oocyte vitrification. High cooling rates allow reduction of the concentration of the cryoprotectants used and in this way reduce their toxicity. Furthermore, a reduction in the amount of cryoprotectants required decreases their toxic and osmotic effects. The volume of the vitrification solution is minimized by using special carriers during the vitrification process. These carriers include: the open pulled straw (OPS; Vajta et al., 1998; Chen et al., 2000a,b; Hurtt et al., 2000; Oberstein et al., 2001), the flexipet-denuding pipette (FDP; Liebermann et al., 2002; J. Liebermann and M. J. Tucker, unpublished), micro-drops (Papis et al., 2000), electron microscope copper grids (EM; Hong et al., 1999; Park et al., 1999; Chung et al., 2000; Park et al., 2000), the 
hemi-straw system (Kuwayama and Kato, 2000; Vandervorst et al., 2001), nylon mesh (Matsumoto et al., 2001) and the cryoloop (Lane et al., 1999a,b; Oberstein et al., 2001; Yeoman et al., 2001; J. Liebermann and M. J. Tucker, unpublished). These carriers or vessels have all been used to achieve higher cooling rates. Vitrified mouse (Chen et al., 2000a), bovine (Vajta et al., 1998; Hurtt et al., 2000), equine (Hurtt et al., 2000) and human oocytes can survive cryopreservation by vitrification (Hunter et al., 1995; Chen et al., 2000b; Chung et al., 2000). Good survival, cleavage and blastocyst formation rates after vitrification of human oocytes and multipronuclear zygotes have been reported (Chung et al., 2000; Park et al., 2000; Liebermann et al., 2002), as have successful pregnancies and deliveries after using vitrification techniques and protocols for human oocytes, day 3 embryos and blastocysts (Kuleshova et al., 1999; Yokota et al., 2000; Yoon et al., 2000; El-Danasouri and Selman, 2001; Mukaida et al., 2001; Jelinkova et al., 2002; Selman and El-Danasouri, 2002). Until now, direct comparison of different cooling regimens using the hemistraw system and the cryoloop for human oocytes and embryos has not been reported.

Despite the fact that vitrification protocols for human oocytes, embryos and blastocysts are entering the mainstream of human-assisted reproductive technologies, so far vitrification as a cryopreservation method has had very little practical impact on human assisted reproduction, and oocyte cryopreservation is still considered experimental. However, reports of successfully completed pregnancies after vitrification of oocytes will encourage further research. Clearly, the lower survival rate of oocytes after cryopreservation in general, and vitrification in particular, means alternative approaches must be explored.

In the present study, aged human oocytes that had failed to fertilize and human embryos derived from abnormally fertilized zygotes that showed one pronucleus or three or more pronuclei were used as a model to determine any possible influence arising from variations in the vitrification technique and protocol, and cooling regimen in terms of survival and developmental potential. The aim of the present study was to compare the effects of vitrification using either hemi-straw system or the cryoloop as carrier on the viability of human oocytes (Expt 1) and the developmental potential of day 3 embryos up to the stage of compaction (Expt 2).

\section{Materials and Methods}

\section{Human oocytes and embryos}

The local ethics committee approved this investigation. Inseminated oocytes that had failed to fertilize $(n=928)$, and abnormally fertilized zygotes $(n=266)$ that showed one pronucleus or three or more pronuclei at the time of the fertilization check (for the presence and number of pronuclei) after standard IVF or intracytoplasmic sperm injection (ICSI) were obtained from patients who gave their informed consent. The percentage of degeneration observed in the vitrified oocytes that represented background loss owing to the nature of the oocytes that had failed to fertilize was assessed by collecting data from July to December 2001 from 810 oocytes from IVF procedures as a control group. The rate of degeneration, the rate of atretic oocytes, and the rate of fractured zona, empty zona and dead oocytes $24 \mathrm{~h}$ after insemination were estimated. The rate of degeneration of control oocytes $24 \mathrm{~h}$ after insemination was $1.6 \%$ of the oocytes (13 of 810 ); of the 13 assessed oocytes, about $0.6 \%$ were empty zona and $0.7 \%$ of all oocytes were dead.

The oocytes that had failed to fertilize and abnormally fertilized zygotes were separated from the normally fertilized zygotes and used for vitrification in this study. The selected oocytes and zygotes from different patients were pooled, and then randomly assigned to groups that would undergo either the hemi-straw system or cryoloop vitrification technique. The oocytes or zygotes from the two groups were vitrified simultaneously. Thus, the possible influence of individual patient variation was excluded or minimized and it could be assured that the two experimental groups of oocytes and zygotes were identical in nature and only differed in terms of the vitrification technique used. Afterwards, the warming of the oocytes was also performed simultaneously.

The recovered zygotes were placed for culture in IVC-1 (In-Vitro Care, San Diego, CA), supplemented with 10\% human serum albumin (HSA) in $20 \mu \mathrm{l}$ drops under mineral oil (SAGE BioPharma, Bedminster, $\mathrm{NJ}$ ) at $37^{\circ} \mathrm{C}$ in a humidified atmosphere of $5 \% \mathrm{CO}_{2}$ in air until day 3 . On day 3 , embryos at the 6-8 blastomere stage of development (average number of cells before vitrification in each experimental group was eight) were pooled and simultaneously vitrified. Day 3 embryos with $>50 \%$ fragmentation were omitted from the vitrification procedures. The embryos were warmed simultaneously and then cultured from day 3 to day 4 in CCM (Vitrolife, Gothenburg).

\section{Vitrification and dilution solutions}

Chemicals for precooling, vitrification and dilution were purchased from Sigma (St Louis, MO) and prepared with Dulbecco's phosphate-buffered saline (DPBS) without calcium or magnesium (In-Vitro Care) with 20\% serum substitute supplement (SSS) (Irvine Scientific, Santa Ana, CA). The $0.25 \mathrm{ml}$ straws used for the hemi-straw system were obtained from IVM (L'Aigle), and the cryoloop (20 $\mu \mathrm{m}$ thickness; 0.5-0.7 mm in loop diameter) from Hampton Research (Laguna Niguel, CA).

Before vitrification using the hemi-straw system or cryoloop, the oocytes were placed in DPBS with 20\% SSS at room temperature for $10 \mathrm{~min}$ to cool down. The vitrification procedure was initiated in culture dishes (Nalge Nunc International, Rochester, NY) prepared with one droplet of $100 \mu \mathrm{l}$ holding medium (HM: DPBS with 20\% SSS), one droplet of $100 \mu \mathrm{l}$ pre-cooling solution (VS1) and one droplet 
of $100 \mu$ l vitrification solution (VS2) and an additional $20 \mu \mathrm{l}$ droplet of VS2. A six-well tissue culture plate (Genesis Instruments, Elmwood, Wl) was prepared with $1 \mathrm{ml}$ of each dilution solution for warming. The solutions for dilution after warming were prepared using DPBS with $20 \%$ SSS and different concentrations of sucrose.

\section{Cryoloop vitrification technique}

The vitrification method using the cryoloop was based on the method described by Lane et al. (1999a,b). The oocytes or embryos were placed in VS1, and then transferred into VS2. The nylon loop was dipped into VS2 to create a thin, filmy layer of the VS2 by surface tension on the nylon loop. After a short exposure to VS2, the oocytes or embryos were placed on to the nylon loop pre-loaded with the thin film of VS2, using a flexipet-denuding pipette (end hole $140 \mu \mathrm{m}$; Cook IVF, Spencer, IN;) and kept there by surface tension. A metal insert on the lip of a cryovial enables the use of a stainless steel rod with a small magnet for manipulation of the nylon loop at low temperatures. The loaded nylon loop was plunged into liquid nitrogen, and then screwed into the cryovial that had previously been submerged in liquid nitrogen, using the stainless steel rod. The vials were attached in standard canes for storage in liquid nitrogen.

\section{Hemi-straw system vitrification technique}

The hemi-straw system vitrification procedure was reported first by Vandervorst et al. (2001). The end of a $0.25 \mathrm{ml}$ straw was cut with a sharp scalpel, so that the end was open (about $1 \mathrm{~cm}$ in length), and it was easy to pipette a small droplet $(<1.0 \mu \mathrm{l})$ onto the open inner face of the straw. The oocytes or embryos were pretreated briefly with VS1. After pre-equilibrating, the oocytes or embryos were placed in VS2, and then transferred with a minimum amount of VS2 into the $20 \mu \mathrm{l}$ droplet of VS2, and loaded in as small a volume as possible $(<1.0 \mu \mathrm{l})$ on to the inner surface of the open edge of the $0.25 \mathrm{ml}$ straw. The straw was then plunged vertically into liquid nitrogen, and placed into a prelabelled $0.5 \mathrm{ml}$ cryostraw (Cryo Bio Systems, L'Aigle) held under liquid nitrogen. The cryostraw was then plugged with a plastic colour-coded plug (Cryo Bio Systems) before cryostorage.

\section{Experimental design}

In pre-trials, the influence of the temperature of the vitrification solution during the loading process $\left(25^{\circ} \mathrm{C}\right.$ versus $37^{\circ} \mathrm{C}$ ) on the survival potential of the oocytes was tested (J. Liebermann and M. J. Tucker, unpublished). It was decided for all protocols to use a loading temperature near room temperature $\left(25^{\circ} \mathrm{C}\right)$. In all protocols, oocytes or embryos were vitrified by the use of a two-step loading process with cryoprotectants. The protocols with the concentrations and properties of each vitrification solution, and the time spent in each solution, are summarized (Table 1).

\section{Experiment 1: oocyte vitrification protocol}

After cooling for $10 \mathrm{~min}$, oocytes were first equilibrated in VS1 containing a mixture of $10 \%$ ethylene glycol, $10 \%$ $(\mathrm{v} / \mathrm{v})$ dimethyl sulphoxide (DMSO) and $1 \mathrm{mg}$ polyethylene glycol ml-1 (average molecular weight 8000 ) for $60 \mathrm{~s}$ at $25^{\circ} \mathrm{C}$ and vitrified by exposure for $20 \mathrm{~s}$ to vitrification solution (VS2) containing 20\% ethylene glycol, 20\% (v/v) DMSO $1 \mathrm{mg}$ polyethylene glycol ml-1, $10 \mathrm{mg}$ Ficoll ml-1 (average molecular weight 400000 ) and 0.65 mol sucrose $\mathrm{ml}^{-1}$.

After storage for 10-14 days, the straws and cryoloops were taken out of the liquid nitrogen. The cryoprotectants were removed by warming the oocytes and diluting them using a four-step dilution with sucrose on a stage at $37^{\circ} \mathrm{C}$. In brief, warming was carried out at $37^{\circ} \mathrm{C}$ by submerging the oocytes in $1 \mathrm{~mol}$ sucrose $\mathrm{I}^{-1}$. After $2 \mathrm{~min}$, oocytes were transferred to $0.5 \mathrm{~mol}$ sucrose $\mathrm{I}^{-1}$ for $3 \mathrm{~min}$, and then to $0.25 \mathrm{~mol}$ sucrose $\mathrm{I}^{-1}$ for $5 \mathrm{~min}$ and finally to $0.125 \mathrm{~mol}$ sucrose $\mathrm{I}^{-1}$ for a further $5 \mathrm{~min}$. After warming, the recovered oocytes were placed in culture in IVC-1 for $24 \mathrm{~h}$, and then evaluated for cryosurvival.

\section{Experiment 2: embryo vitrification protocols}

Embryos were first equilibrated in VS1 containing a mixture of $10 \%$ EG and $10 \%(\mathrm{v} / \mathrm{v})$ DMSO for 4 min at $25^{\circ} \mathrm{C}$ and vitrified after exposure for $20 \mathrm{~s}$ to $20 \%$ ethylene glycol,

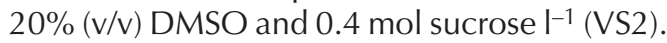

After storage for 10-14 days, the embryos in both carriers were simultaneously warmed and diluted using a three-step dilution with sucrose on a stage at $37^{\circ} \mathrm{C}$. In brief, embryos were submerged in a solution of 1 mol sucrose ${ }^{-1}$. After $5 \mathrm{~min}$, they were transferred into $0.25 \mathrm{~mol}^{-}$sucrose $\mathrm{I}^{-1}$ for $3 \mathrm{~min}$, and finally into $0.125 \mathrm{~mol}^{\text {sucrose }} \mathrm{I}^{-1}$ for $2 \mathrm{~min}$.

\section{Evaluation of the viability of vitrified-warmed human oocytes and embryos}

After warming, the recovered oocytes and embryos were placed in $20 \mu \mathrm{l}$ drops under mineral oil at $37^{\circ} \mathrm{C}$ in a humidified atmosphere of $5 \% \mathrm{CO}_{2}$ in air for the next $24 \mathrm{~h}$. IVC-1 was used as culture medium for the oocytes, whereas the embryos were incubated in CCM.

After $24 \mathrm{~h}$, oocytes were evaluated for cryosurvival. The number of oocytes with fragmented cytoplasm, indistinct oolemma or damaged zona pellucida, and those that degenerated during the $24 \mathrm{~h}$ culture period were recorded as not surviving after vitrification.

Two hours after warming, embryos were assessed to determine their integrity and the number of surviving blastomeres. Surviving embryos were defined as having half or more of their blastomeres intact after warming. Therefore, the survival rate was judged as the percentage of embryos that were morphologically intact $(\geqslant 50 \%$ intact blastomeres) after removal of the cryoprotectant. In addition, after culture for $24 \mathrm{~h}$, the embryos were checked for further cleavage and compaction. 
Table 1. Summary of the protocols of each experiment regarding concentration and properties of the vitrification solution, time in each solution, carrier used and type of oocyte examined

\begin{tabular}{|c|c|c|c|c|}
\hline & Holding medium (HM) & Vitrification solution 1 (VS1) & Vitrification solution 2 (VS2) & Carrier \\
\hline Oocytes & $\begin{array}{c}20 \% \text { SSS in DPBS } \\
\text { (10 min })\end{array}$ & $\begin{array}{c}10 \% \text { EG, } 10 \% \text { DMSO, } \\
1 \mathrm{mg} \mathrm{PEG} \mathrm{ml}^{-1} \\
(60 \mathrm{~s})\end{array}$ & $\begin{array}{c}20 \% \text { EG, } 20 \% \mathrm{DMSO}^{-} \\
1 \mathrm{mg} \mathrm{PEG} \mathrm{m}^{-1}, \\
10 \mathrm{mg} \mathrm{Ficoll} \mathrm{m}^{-1}, \\
0.65 \mathrm{~mol} \mathrm{sucrose}^{-1}(30 \mathrm{~s})\end{array}$ & $\mathrm{CL} / \mathrm{HSS}$ \\
\hline Embryos & - & $\begin{array}{c}10 \% \text { EG, } 10 \% \text { DMSO } \\
(4 \mathrm{~min})\end{array}$ & 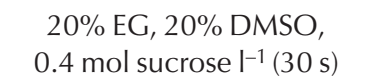 & $\mathrm{CL} / \mathrm{HSS}$ \\
\hline
\end{tabular}

DPBS: Dulbecco's phosphate-buffered saline; SSS: serum substitute supplement; EG: ethylene glycol; DMSO: dimethyl sulphoxide; PEG: polyethylene glycol; CL: cryoloop; HSS: hemi-straw system.

Table 2. Survival after warming of human oocytes after $24 \mathrm{~h}$ culture in cryoloop or hemi-straw system

\begin{tabular}{lcc}
\hline & \multicolumn{2}{c}{ Vitrification technique } \\
\cline { 2 - 3 } & Cryoloop & Hemi-straw system \\
\hline Number of oocytes & 448 & 480 \\
Oocyte survival (\%) & $361(80.6 \%)^{*}$ & $410(85.4 \%)^{*}$ \\
Overall survival (\%) & 771 of $928(83.0 \%)$ \\
\hline
\end{tabular}

${ }^{*} P=0.07$, not significant, chi-squared test comparison of cryoloop versus hemi-straw system

\section{Statistical analysis}

Statistical analysis was carried out by means of a chisquared test. Statistical significance was defined as $P<0.05$.

\section{Results}

The survival rates of oocytes after warming using the cryoloop or the hemi-straw system are shown (Table 2). In Expt 1, 928 aged human oocytes that had failed to fertilize were subjected to vitrification. The rate of survival of oocytes after $24 \mathrm{~h}$ of culture was slightly lower in the cryoloop group than in the hemi-straw system group ( $80.6 \%$ versus $85.4 \%)$, but the difference was not significant. Overall $83.0 \%$ of oocytes survived (771 of 928).

The survival rate after warming of day 3 embryos with more than half of the blastomeres intact was slightly lower in the cryoloop group (83.8\%) compared with the survival in the hemi-straw system group $(89.7 \%)$, but the difference was not significant (Table 3). In general, the survival rate of vitrified embryos after $2 \mathrm{~h}$ of culture was $86.8 \%$ (231 of 266). A reasonable rate of further cleavage and compaction occurred in the cryoloop group (29.4\%; 32 of 109), which was significantly different from that in the hemi-straw system group $\left(37.7 \% ; 46\right.$ of $122 ; \chi^{2} ; P=0.002$; Table 3$)$. Overall, 78 of 231 of the day 3 embryos became compacted (33.8\%).

\section{Discussion}

Vitrification, as a simple cryopreservation method of directly submerging a cell in liquid nitrogen after brief exposure to a cryoprotectant agent, is substantially less
Table 3. Survival after warming of human day 3 embryos after $2 \mathrm{~h}$ culture, and cleavage behaviour after $24 \mathrm{~h}$ using cryoloop and hemi-straw system

\begin{tabular}{lcc}
\hline & \multicolumn{2}{c}{ Vitrification technique } \\
\cline { 2 - 3 } & Cryoloop & Hemi-straw system \\
\hline $\begin{array}{l}\text { Number of embryos } \\
\begin{array}{c}\text { Survival }(\geqslant 50 \% \text { of } \\
\text { intact blastomeres) }\end{array}\end{array}$ & 130 & 136 \\
$\begin{array}{c}\text { Overall embryo survival } \\
(\geqslant 50 \% \text { of intact }\end{array}$ & $109(83.8 \%)^{*}$ & $122(89.7 \%)^{*}$ \\
blastomeres) & & \\
$\begin{array}{c}\text { Day } 4 \text { ( } \geqslant 8 \text { blastomeres and } \\
\text { stage of compaction; } \%)\end{array}$ & $32(29.4 \%)^{* *}$ & $46(37.7 \%)^{* *}$ \\
Overall compaction rate $(\%)$ & 78 of $231(33.8 \%)$ \\
\hline
\end{tabular}

$* P=0.07$, not significant, chi-squared test comparison of cryoloop versus hemi-straw system (survival); ${ }^{* *} P=0.002$, chi-squared test comparison of cryoloop versus hemi-straw system (development potential).

harmful to cells such as oocytes and embryos that have high chilling sensitivities than are conventional cryopreservation methods using traditional slow-freezing (Martino et al., 1996; Vatja et al., 1997). The advantage for the cells of vitrification over conventional cryopreservation procedures is that the 'open system' lacks an insulating layer around the sample. The vapour surrounding the cells can create effective insulation that reduces temperature transfer, resulting in a decreased cooling rate and possibly crystallization. The volume of the vitrification solution should be minimized as much as is practical to ensure that the cells are surrounded with liquid nitrogen and not vapour. Thus, the duration of any coating vapour is reduced and the rate of cooling is increased. However, the direct and rapid contact with liquid nitrogen, coupled with as small a volume of the vitrification solution as is practical, allows higher cooling rates to be achieved, because conductive heat transfer in liquid is much faster than it is in vapour. Furthermore, the high rate of cooling has the positive effect of reducing the concentration of cryoprotectant and the exposure time. Thus, exposure to the toxic effects of the cryoprotectants is also decreased (J. Liebermann and M. J. Tucker, unpublished).

The results of the present study indicate a high rate of 
survival after warming of morphologically normal aged oocytes that had failed to fertilize and day 3 embryos when vitrification protocols with high rates of cooling are used. Furthermore, very promising results were obtained using a mixture of $20 \%$ ethylene glycol and $20 \%(\mathrm{v} / \mathrm{v})$ DMSO. Although human embryo cryopreservation has become a well-established process in assisted human reproduction, oocyte cryopreservation, regardless of some success with this approach (Van Uem et al., 1987; Chen, 1988; Polak et al., 1998; Tucker et al., 1998a,b; Yang et al., 1998), is still considered experimental, and is thought to be of limited clinical use. Unlike all stages of human preimplantation embryos, oocytes are more vulnerable to the cryopreservation procedures involving ice crystallization because of the reduced permeability of the cytoplasmic membranes of oocytes (Ruffing et al., 1993). In addition, cryopreserved cells just after warming are more sensitive to osmotic swelling than are fresh cells (Pedro et al., 1997).

Conventional cryopreservation protocols for human oocytes have resulted in various types of injury (for example, damage of intracellular lipid droplets, and the cytoskeleton), leading to low overall rates of success of about 1\% implantation per egg frozen (Mandelbaum et al., 1998). In addition, the developmental potential of 'slowrate' frozen human oocytes after fertilization, up to cavitation and blastocyst formation, is only $5.6 \%$ (Cobo et al., 2001). In contrast, a recent study showed the beneficial effect of microinjected trehalose on the survival of human oocytes after conventional cryopreservation (Eroglu et al., 2002 ). About $66 \%$ of oocytes in the intracellular trehalose group survived cooling to $-60^{\circ} \mathrm{C}$, whereas all oocytes in the control group degenerated when cooled to $-60^{\circ} \mathrm{C}$. However, similar survival rates (approximately $65 \%$ ) have been reported after vitrification of human oocytes using high concentrations of cryoprotectant agent (Hunter et al., 1995; Kuleshova et al., 1999; Chen et al., 2000b). Kuwayama and Kato (2000) described a minimum volume cooling (MVC) technique that is comparable to the hemi-straw system used in the present study. With a small number of human oocytes, a high survival rate after warming of $91 \%$ (60 of 64) was achieved by using 30 or $40 \%$ ethylene glycol +1.0 or 0.5 mol sucrose $\mathrm{I}^{-1}$ (Kuwayama and Kato, 2000). Vitrification of human oocytes that had failed to fertilize using the cryoloop and a mixture of $20 \%$ ethylene glycol and $20 \%(\mathrm{v} / \mathrm{v})$ DMSO without added macromolecules such as polymers resulted in survival after warming of $81.1 \%$ (574 of 708; J. Liebermann and M. J. Tucker, unpublished) indicating that increased rates of cooling are beneficial for oocyte vitrification. It has been reported previously that increased rates of cooling result in more successful vitrification of animal oocytes (Arav et al., 2000; Hochi et al., 2001; Isachenko et al., 2001).

Experience with carriers such as flexipet-denuding pipette (FDP) with or without liquid nitrogen slush (Liebermann et al., 2002) and cryoloop led the present authors to search for new types of carrier. It was necessary to find materials with rapid heat transfer that also support the process of uniform heat exchange. The hemi-straw system has been used in only a few studies and with a small number of cells, but still with very good results. This system is very simple, allowing a very fast rate of cooling by direct contact with liquid nitrogen; it is easy to handle for any embryologist; it is low cost and easy to store in any commercially available storage system (Vandervorst et al., 2001). In contrast to the nylon loop technique, in which the oocytes or embryos are vitrified by placing them on to a thin film on a small nylon loop, with the hemi-straw system, the oocytes or embryos are placed in a very small droplet $(\leqslant 1.0$ $\mu \mathrm{l})$ on an open edge of a cut $0.25 \mathrm{ml}$ cryostraw. The results of Expt 1 of the present study demonstrate that the hemistraw system and the cryoloop both have a beneficial effect on survival after warming, and achieve consistent rates of survival of between 80 and $85 \%$. Furthermore, the high rate of cooling attained using the hemi-straw system or cryoloop allows an apparently beneficial reduction in the concentration of the cryoprotectant. Kuleshova et al. (1999) achieved a rate of survival after warming of human oocytes of approximately $65 \%$, using the OPS and $40 \%$ ethylene glycol as vitrification solution. In the present study, $40 \%$ ethylene glycol was replaced by a mixture of $20 \%$ ethylene glycol and $20 \%(\mathrm{v} / \mathrm{v})$ DMSO. Thus the toxicity of the cryoprotectant was reduced, possibly accounting for the higher survival rate after using the cryoloop or the hemi-straw system for vitrification. Results of the present study indicate that using the hemi-straw system has a more positive effect on the survival after warming than using the cryoloop. One reason for this may be that cells loaded on the carrier build a more effective viscous matrix for encapsulation of these cells in the hemi-straw system, and also prevent crystallization during cooling and warming. These observations were supported by Kuwayama and Kato (2000), who reported three efficient vitrification techniques, one of which they recommended for clinical use: the vitrification technique of placing a fine drop on the inner surface of a straw, similar to the method used for hemi-straw system in the present study.

Reports of human embryo vitrification are rare. ElDanasouri and Selman (2002) reported successful pregnancies and deliveries after vitrification of day 3 human embryos using the OPS. The structure of the OPS covers the vitrification solution containing the embryos, so that contact between the vitrification solution and the liquid nitrogen is not as direct as when using the cryoloop or hemi-straw system. The results showed a negative correlation between stage of development and survival: eight-cell embryos showed a higher survival rate $(79.2 \% ; 62$ of 78$)$ than did embryos with fewer than six cells $(21.1 \%$; 11 of 53$)$ after vitrification (El-Danasouri and Selman, 2002). The results recorded in Expt 2 of the present study show a survival rate of human day 3 embryos with an average of eight blastomeres of between 84 and $90 \%$. This finding supports previous reports in which high survival rate of eight-cell human embryos using $40 \%$ ethylene glycol were documented (Otha et al., 1996; Mukaida et al., 1998). Nevertheless, it is important to note that in the present study the ethylene 
glycol concentration was only $20 \%$, combined with $20 \%$ $(\mathrm{v} / \mathrm{v})$ DMSO. It is possible that this improved success is a result of a higher cooling rate obtained by increasing the speed of thermal conduction using the cryoloop or the hemi-straw system. Despite the fact that a promising rate of survival after warming was achieved, overall only about $34 \%$ of the surviving embryos had the developmental potential to reach the stage of compaction. Significantly more embryos compacted in the hemi-straw system group than in the cryoloop group. The morphological grade of the embryos was not dependent on the carrier system used. The findings of the present study support a previous report (Kuwayama and Kato, 2000) in which significantly higher rates of cleavage and blastocyst formation of bovine embryos were obtained with the MVC method than using plastic straws and open pulled straws. However, the compaction rate of day 3 embryos after warming reported in this study needs to be improved, although it should be noted that these embryos were obtained after abnormal fertilization, and may not have the same cryobiological properties and potential for development as embryos obtained after normal fertilization (Noto et al., 1991; Joris et al., 1999).

In conclusion, the present study demonstrates that using the cryoloop or the hemi-straw system, which enabled ultra-rapid cooling rates to be achieved, in combination with a low concentration of a mixture of ethylene glycol and DMSO as cryoprotectant agents can effectively improve survival rates of human oocytes and day 3 embryos after warming after vitrification. The hemi-straw system may be a superior method to the cryoloop, particularly as it achieves a higher rate of compaction for day 3 embryos after warming. The vitrification techniques described here appear to be a good alternative to classical slow-freezing protocols. Therefore, the results presented in this study are encouraging, and, together with the more convenient protocols of vitrification in general, should generate further research to develop this technique and increase its clinical use and efficiency.

\section{References}

Arav A and Zeron Y (1997) Vitrification of bovine oocytes using modified minimum drop size technique (MDS) is affected by the composition and concentration of the vitrification solution and by the cooling conditions Theriogenology 47341 (Abstract)

Arav A, Zeron Y and Ocheretny A (2000) A new device and method for vitrification increases the cooling rate and allows successful cryopreservation of bovine oocytes Theriogenology 53248 Abstract

Chen C (1988) Pregnancies after human oocyte cryopreservation Annals of the New York Academy of Sciences $\mathbf{5 4 1} 541-549$

Chen SU, Lien YR, Chen HF, Chao KH, Ho HN and Yang YS (2000a) Open pulled straws for vitrification of mature mouse oocytes preserve patterns of meiotic spindles and chromosomes better than conventional straws Human Reproduction 15 2598-2603

Chen SU, Lien YR, Chao KH, Lu HF, Ho HN and Yang YS (2000b) Cryopreservation of mature human oocytes by vitrification with ethylene glycol in straws Fertility and Sterility $\mathbf{7 4} 804-808$

Chung HM, Hong SW, Lim JM, Lee SH, Cha WT and Ko JJ (2000) In vitro blastocyst formation of human oocytes obtained from unstimulated and stimulated cycles after vitrification at various maturational stages Fertility and Sterility 73 545-551
Cobo A, Rubio C, Gerli S, Ruiz A, Pellicer A and Remohi J (2001) Use of fluorescence in situ hybridization to assess the chromosomal status of embryos obtained from cryopreserved oocytes Fertility and Sterility $\mathbf{7 5}$ 354-360

El-Danasouri I and Selman H (2001) Successful pregnancies and deliveries after a simple vitrification protocol for day 3 human embryos Fertility and Sterility $\mathbf{7 6}$ 400-402

Eroglu A, Toner M and Toth TL (2002) Beneficial effect of microinjected trehalose on the cryosurvival of human oocytes Fertility and Sterility 77 152-158

Fahy GM, McFarlane DR, Angell CA and Meryman HT (1984) Vitrification as an approach to cryopreservation. Cryobiology 21 407-426

Hochi S, Akiyama M, Minagawa G, Kimura K and Hanada A (2001) Effects of cooling and warming rates during vitrification on fertilization of in vitro-matured bovine oocytes Cryobiology 42 69-73

Hong SW, Chung HM, Lim JM et al. (1999) Improved human oocyte development after vitrification: a comparison of thawing methods Fertility and Sterility 72 142-146

Hunter JE, Fuller BJ, Bernard A, Jackson A and Shaw RW (1995) Vitrification of human oocytes following minimal exposure to cryoprotectants; initial studies on fertilization and embryonic development Human Reproduction 10 1184-1188

Hurtt AE, Landim-Alvarenga F, Seidel GE, Jr and Squires EL (2000) Vitrification of immature and mature equine and bovine oocytes in an ethylene glycol, ficoll and sucrose solution using open-pulled straws Theriogenology 54 119-128

Isachenko V, Alabart JL, Nawroth F, Isachenko E, Vajta G and Folch J (2001). The open pulled straws vitrification of ovine GV-oocytes: positive effect of rapid cooling or rapid thawing or both? CryoLetters 22 157-162

Jelinkova L, Selman HA, Arav A, Strehler E, Reeka N and Sterzik K (2002) Twin pregnancy after vitrification of 2-pronuclei human embryos Fertility and Sterility 77 412-414

Joris H, Van den Abbeel E, De Vos A and Van Steirteghem A (1999) Reduced survival after human embryo biopsy and subsequent cryopreservation Human Reproduction 14 2833-2837

Kuleshova L, Gianaroli L, Magli C, Ferraretti A and Trounson A (1999) Birth following vitrification of a small number of human oocytes: case report Human Reproduction 14 3077-3079

Kuwayama M and Kato O (2000) Successful vitrification of human oocytes Fertility and Sterility $\mathbf{7 4}$ (Supplement 3) S49 Abstract O-127

Lane M, Schoolcraft WB and Gardner DK (1999a) Vitrification of mouse and human blastocysts using a novel cryoloop container-less technique Fertility and Sterility 72 1073-1078

Lane M, Bavister BD, Lyons EA and Forest KT (1999b) Container-less vitrification of mammalian oocytes and embryos Nature Biotechnology 17 1234-1236

Liebermann J, Tucker M, Graham J, Han T, Davis A and Levy M (2002) Blastocyst development after vitrification of multipronucleate zygotes using the flexipet denuding pipette (FDP) Reproductive BioMedicine Online 4 146-150

Mandelbaum J, Belaisch-Allart J, Junca AM et al. (1998) Cryopreservation in human assisted reproduction is now routine for embryos but remains a research procedure for oocytes Human Reproduction 13 (Supplement 3) $161-174$

Martino A, Songsasen N and Leibo SP (1996) Development into blastocysts of bovine oocytes cryopreserved by ultra-rapid cooling Biology of Reproduction 54 1059-1069

Matsumoto H, Jiang JY, Tanaka T, Sasada H and Sato E (2001) Vitrification of large quantities of immature bovine oocytes using nylon mesh Cryobiology 42 139-144

Mukaida T, Wada S, Takahashi K, Pedro PB, An TZ and Kasai M (1998) Vitrification of human embryos based on the assessment of suitable conditions for 8-cell mouse embryos Human Reproduction 13 2874-2879

Mukaida T, Nakamura S, Tomiyama T, Wada S, Kasai M and Takahashi K (2001) Successful birth after transfer of vitrified human blastocysts with use of a cryoloop containerless technique Fertility and Sterility $\mathbf{7 6}$ 618-623 
Noto V, Campo R, Roziers P and Gordts S (1991) Fluorescein diacetate assessment of embryo viability after ultra rapid freezing of human multipronucleate embryos Fertility and Sterility 55 1171-1175

Oberstein N, O'Donovan MK, Bruemmer JE, Seidel GE, Jr, Carnevale EM and Squires EL (2001) Cryopreservation of equine embryos by open pulled straws, cryoloop, or conventional cooling methods Theriogenology 15 607-613

Ohta N, Nohara M and Kojimahara T (1996) Ultrarapid freezing of human embryos by vitrification Japanese Journal of Fertility and Sterility $\mathbf{4 1}$ 276-279

Papis K, Shimizu M and Izaike Y (2000) Factors affecting the survivability of bovine oocytes vitrified in droplets Theriogenology 15 651-658

Park SP, Kim EY, Oh JH et al. (2000) Ultra-rapid freezing of human multipronuclear zygotes using electron microscope grids Human Reproduction 15 1787-1790

Pedro PB, Zhu SE, Makino N, Sakurai T, Edashige K and Kasai M (1997) Effects of hypotonic stress on the survival of mouse oocytes and embryos at various stages Cryobiology 35 150-158

Polak de Fried E, Notrica J, Rubinstein M, Marazzi A and Gomez Gonzalez M (1998) Pregnancy after human donor oocyte cryopreservation and thawing in association with intracytoplasmic sperm injection in a patient with ovarian failure Fertility and Sterility $69555-557$

Rall WF (1987) Factors affecting survival of mouse embryos cryopreserved by vitrification Cryobiology 24 387-402

Rall WF and Fahy GM (1985) Ice-free cryopreservation of mouse embryos at $-196^{\circ} \mathrm{C}$ by vitrification Nature $313573-575$

Ruffing NA, Steponkus PL, Pitt RE and Parks JE (1993) Osmometric behavior, hydraulic conductivity, and incidence of intracellular ice formation in bovine oocytes at different developmental stages Cryobiology 30 562-580

Selman HA and El-Danasouri I (2002) Pregnancies derived from vitrified human zygotes Fertility and Sterility 77 422-423

Tucker MJ, Morton PC, Wright G, Sweitzer CL and Massey JB (1998a) Clinical application of human egg cryopreservation Human Reproduction 13 3156-3159
Tucker MJ, Wright G, Morton PC and Massey JB (1998b) Birth after cryopreservation of immature oocytes with subsequent in vitro maturation Fertility and Sterility 70 578-579

Vajta G, Booth PJ, Holm P, Greve T and Callesen H (1997) Successful vitrification of early stage bovine in vitro produced embryos with the Open Pulled Straw (OPS) method CryoLetters 18 191-195

Vajta G, Holm P, Kuwayama M et al. (1998). Open Pulled Straws (OPS) vitrification: a new way to reduce cryoinjuries of bovine ova and embryos Molecular Reproduction and Development 51 53-58

Vandervorst M, Vanderzwalmen P, Standaart V et al. (2001) Blastocyst transfer after vitrification in a hemi-straw (HS) system Human Reproduction 16 (Abstract book 1) 153-154 Abstract P-133

Van Uem JFHM, Siebzehnrubl ER, Schuh B, Koch R, Trotnow S and Lang N (1987) Birth after cryopreservation of unfertilized oocytes Lancet $\mathbf{i}$ $752-753$

Yang DS, Winslow KL and Blohm PL (1998) Improved survival rate after cryopreservation of human fresh and aged unfertilized oocytes using a specially developed oocyte cryopreservation regime Fertility and Sterility 70 (Supplement 1) S86 Abstract O-232

Yeoman RP, Gerami-Naini B, Mitalipov S, Nusser KD, Widmann-Browning AA and Wolf DP (2001) Cryoloop vitrification yields superior survival of Rhesus monkey blastocysts Human Reproduction 16 1965-1969

Yokota Y, Sato S, Yokota M et al. (2000) Successful pregnancy following blastocyst vitrification Human Reproduction 15 1802-1803

Yoon TK, Chung HM, Lim JM, Han SY, Ko JJ and Cha KY (2000) Pregnancy and delivery of healthy infants developed from vitrified oocytes in a stimulated in vitro fertilization-embryo transfer program [letter] Fertility and Sterility 74 180-181

Received 22 April 2002.

First decision 31 May 2002.

Revised manuscript received 17 June 2002.

Accepted 20 June 2002. 